\title{
Word associations to compound stimuli under two presentational methods'
}

BARBARA S. MUSGRAVE AND JEAN CARL COHEN

SMITH COLLEGE

Two presentational methods were used to gather word associations to four-word stimuli consisting of three context words preceding a test word. According to other one-word stimulus norms, all four words of the compound elicit the same primary response, the desired response (DR). Method 1 (auditory, E-paced, and presenting each four-word stimulus as a temporal sequence) was significantly superior to Method 2 (visual, S-paced, and exposing the four stimulus words simultaneously) in increasing $D R$ elicitation and decreasing number of difference responses.

Word association (WA) studies have presented compound stimuli in a variety of ways. Both visual and auditory modes have been used. The task has been paced or unpaced. Many other methodological aspects, such as instructions, list length, extent of materials exposed simultaneously, whether the number of words in each stimulus is the same or mixed, and the number of times a particular word or its high associates appeared in different stimuli in the list, have been varied concomitantly. On occasion, data for control and experimental groups have been gathered under different presentational methods.

The present study is not parametric concerning these many variables, but is simply a comparison of two different composite methods. They differ on three principal dimensions: sensory mode, $E$ vs $S$ control of pacing, and sequential vs simultaneous exposure of the words in a stimulus compound. Each variation of these dimensions has been used with some frequency in the past (e.g., Cramer, 1964; Howes \& Osgood, 1954; Jenkins \& Cofer, 1957; Musgrave, Cohen, \& Robbins, in press), and a finding of differing results would have interesting theoretical as well as methodological implications.

Method

One hundred underclass college women, native English speakers, naive to WA experiments, were divided into two groups on arrival for class at seven sections of an introductory experimental psychology course. The two conditions were run simultaneously for each section in separate rooms with equal groups of four to eight Ss. Each $S$ was given a 29 page, 5-1/2 $\times 3$ in. booklet with a cover sheet on which to record the experiment's start and stop times, and 28 pages on which to write responses, one to a page.

The 28 compound stimuli each consisted of four words. All four words elicited the same primary response, the DR for the four word compound, according to WA norms gathered elsewhere to visually presented one word stimuli. The norms showed that only a few words elicited as a response any other word within the same compound stimulus, elicitations being of extremely low frequency, if not idiosyncratic. In addition, very few words elicited any other word on the list with appreciable frequency. The DR for each of the $\mathbf{2 8}$ compound stimuli was unique, thus automatically was not the primary of any stimulus word other than its own four, and was ellcited as a lower strength response by only an occasional word in any other compound than its own. Because of differences in the normative frequency of the DRs to the single words, the four words of each compound were assigned randomly to positions within the compound, with the overall restriction that the mean DR frequencies for the four positions across the 28 compound stimuli should be approximately equal. Each of the 112 stimulus words appeared exactly once in the list.

Method 1 (M-1) presented the stimuli aurally by tape recorder with each four word set allotted approximately $5 \mathrm{sec}$, and with a 5-6 sec response interval between offset of one four word compound and onset of the next. M-1 was, therefore, paced by $E$, and each stimulus word in the sequence terminated as spoken. The last word of each compound, the test word, was spoken in a lowered tone, with finality. Method 2 (M 2) presented one four word stimulus per page, in upper case letters, with one space between words, in a horizontal line, with the test word last reading left to right. Hence, all four words of a stimulus under M-2 were exposed simultaneously, and constantly until the page was turned. $\mathrm{M-2}$ Ss were instructed to read the four words from left to right, and to work rapidly. But progress from word to word within a compound stimulus, and from compound to compound page by page, was controlled and paced by each $\mathrm{S}$.

As to methodological uniformities across the two conditions: The same stimuli were presented, in the same three different list orders. Ss were instructed to listen to $(M-1)$, or read from left to right (M-2) all four words in each stimulus and associate to the last word, to write the first other word thought of, to write only one word as a response to each compound, and to write on each page in order. For $\mathrm{M}-2$, underlined response blanks followed each four word stimulus in the same horizontal line; for $M-1$, they appeared alone in the center of each page.

Results and Discussion

All DRs were primaries to the compound stimuli except for one compound under M-2 when the DR was secondary. M-1 elicited the DR more frequently for 
23 of the 28 stimuli than did $\mathrm{M}-2$, and tied with $\mathrm{M}-2$ in three additional cases. Mean DR frequency for M-1 was 29.79 (out of a possible score of 50), with $\mathrm{SD}=8.34$; for $\mathrm{M}-2$ the mean was 23.82 , with $\mathrm{SD}=9.27$ $(t=5.92, d f=27, p<.001)$. The mean number of different responses for $\mathrm{M}-1,12.96$ with an SD of 3.93, was less than that for M-2, which was 15.93 with an $\mathrm{SD}$ of $5.01(t=3.55, \mathrm{df}=27, \mathrm{p}<.01)$. Thus, $M-1$ was significantly superior to $\mathrm{M}-2$ in producing $D R s$, and decreasing number of different responses.

The response hierarchy of each stimulus, plotted as a frequency distribution (number of responses given by only one S, by two Ss, etc.) was cumulated across the 28 stimuli for each method. Both composite distributions were so skewed as to be $L$ shaped, descending steeply from high numbers of responses given by one $S$ and by two Ss, and continuing as long, thin, fairly flat tails extending to the highest commonality responses, those given by 45 or $46 \mathrm{Ss}$. In the tails, responses given by 11 Ss up to 46 Ss were chiefly DR primaries. The $M-2$ distribution for primaries was thicker in the 11-23 range than that for $M-1$, and thinner thereafter. In the 1-11 range, the only marked difference between the distributions was that the frequency of idiosyncratic and lowest commonality responses was considerably higher under M-2 than M-1.

Inasmuch as the two methods differed in at least three dimensions, further studies are needed to evaluate the contribution of each methodological difference. Difference in mode may contribute in several ways. For example, it may affect responding to the single words in each compound. For children, auditory, compared with visual, presentation of single word stimuli facilitates elicitation of high commonality responses (Palermo, 1965). This may hold for adults also (see Jenkins \& Palermo, 1965, p. 309).

pacing, and sequential vs simultaneous exposure, may be closely related factors, having quite similar effects on such underlying mechanisms as short-term memory. M-1 Ss, with each word of the four terminating as spoken, may have been under pressure to respond quickly while memory of the words was strongest, as well as under pressure to get ready for the inexorable onset of four more briefly presented words. Observably, many $M-1$ ss turned to the next blank page appreciably before the onset of the next stimulus, thus creating a stimulus-free interval which may have served to diminish any effects of preceding words. In contrast, M-2 Ss, although told to work rapidly, knowing total task time was being recorded, and actually completing the task in less time on the average ( 4.13 vs $5 \mathrm{~min}$ ), were under no compulsion to finish with each stimulus rapidiy either to avoid forgetting it, or to avoid missing the next stimulus. Perhaps for these reasons, the pacing of M-2 Ss was observably different, with the offset of each four word stimulus and its written response followed immediately, at the flip of a page, by the onset of the next four word stimulus. This kind of pacing may have produced, at stimulus onset, much higher levels of availability for much larger numbers of potential responses than occurred under M-1 with its offsetonset intervals.

Thus, WA phenomena seem a sensitive kind of performance, worthy of more fine grained exploration than they have received. Surely, it is clear that until further studies clarify the contribution of mode, E-S pacing, and sequential vs simultaneous presentation, all three of these dimensions should be considered potential sources of difference in comparisons between studies, or between experimental and control groups within a single study.

References

CRAMER, PHEBE Successful mediated priming via associative bonds. Psychol. Rep., 1964, 15, 235-238.

HOWES, D., \& OSGOOD, C. E. On the combination of associative probabilities in linguistic contexts. Amer. J. Psychol, 1954, 67, 241-258.

JENKINS, J. J., \& PALERMO, D. S. Further data on changes in wordassociation norms. J. Pers. soc. Psychol, 1965, 1, 303-309.

JENKINS, PATRICIA M. \& COFER, C. N. An exploratory study of discrete free association to compound verbal stimuli. Psychol. Rep., 1957, 3, 599-602.

MUSGRAVE, BARBARA S., COHEN, JEAN C., \& ROBBINS, DONNA M. G. Convergent popular associations in a word-association task. $J$. verbal Learn. verbal Bı/hav., in press.

PALERMO, D. S. Characteristics of word association responses obtained from children in grades one through four. Paper presented at the meetings of the Society for Research in Child Development. Minneapolis, 1965.

Note

1. This study was supported in part by Research Grant MH 08156 from the National Institute of Mental Health. Thanks are due Donna M. G. Robbins and Marilyn Ingellis for aid in gathering and processing data. 